\title{
Entity Name Person Data Type
}

National Cancer Institute

\section{Source}

National Cancer Institute. Entity Name Person Data Type. NCI Thesaurus. Code C95656.

A data type comprised of the name of a person. 